\title{
The Degree of Dysplasia in Neutrophils With A Special Note on Pelger- Huët Anomaly
}

\author{
Ayeesha Sithika Thajudeen* and Priavadhana Rajan Prasaad \\ Department of Pathology, Sree Balaji Medical College \& Hospital, Chennai, India.
}

\section{ABSTRACT}

The Pelger- Huët anomaly (PHA), an autosomal dominant condition ,is characterized by morphologically abnormal neutrophil. This article summarizes the history of PHA, pathogenesis and guidance for differentiating congenital or acquired PHA, with their different morphological variants. We report an interesting case of pseudo-PHA in a 70 year old male diagnosed with Leptospirosis, showing unilobed, unilobed intended, bilobed pelgeroid cells in the peripheral smear.

Keywords: Pelger-Huët Anomaly, Pseudo- Pelger-Huët Anomaly, Dysplastic Neutrophils, Leptospirosis

\section{Introduction}

The polymorphonuclear neutrophils (PMNs) have lobulated nucleus with three to four segments with condensed chromatin. The segmentation helps in diapedesis and chemotaxis of the neutrophil. Pelger- Huët anomaly (PHA) is characterised by hyposegmentation of the neutrophil with bilobed nuclei connected by a thin strand of chromatin. These pelgeroid cells have a characteristic 'prince-nez' appearance. The chromatin is dense and coarser than that of the normal neutrophil. It can be congenital or acquired. The pseudo or acquired Pelger- Huët anomaly occurs in severe infections, use of certain drugs or underlying hematological malignancies like Myelodysplastic syndrome (MDS) and Acute Myeloid Leukemia (AML).$^{[1]}$

\section{Case Report}

A 70 year old gentleman, hailing from South India, came with complaints of intermittent, high grade fever associated with chills and rigor. He was a known case of systemic hypertension on regular treatment. He had no significant family history. Physical examination was unremarkable. Complete blood count showed $\mathrm{Hb}-8.6 \mathrm{~g} / \mathrm{dl}$, leucocytosis with a total count of $25,240 / \mathrm{cu} \mathrm{mm}$ with differential count showing 95\% neutrophils and platelet count was 79,000 cells/cu mm. Peripheral smear examination revealed microcytic hypochromic red blood cells with neutrophilic leucocytosis with shift to left showing band forms, unilobed and bilobed pelgeroid cells with hypogranulation. (Fig 1-4). The table shows the manual differential count. (Table 1). An interesting feature of a phagocytic cell with an engulfed erythrocyte was also noted. (Fig 5).So a diagnosis of pseudo PHA was considered. His workup for pyrexia showed leptospirosis positive with IgM ELISA test. He was started on Doxycycline. The pelgeroid cells on peripheral smear resolved with doxycycline treatment, implying that the changes were most likely reactive manifestations rather than an underlying hematological malignancy.

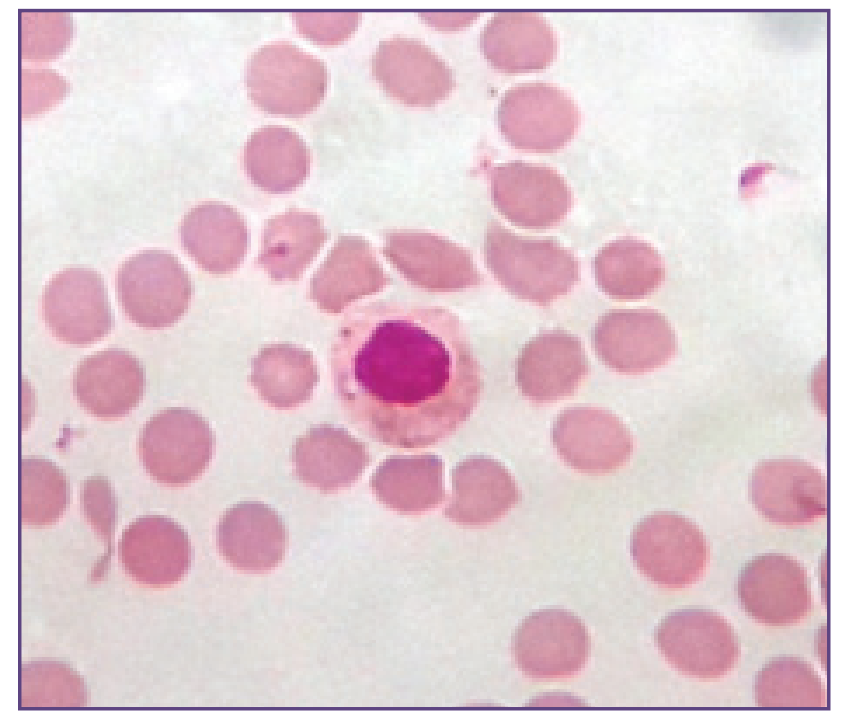

Fig. 1: Neutrophil showing unilobed nucleus with condensed chromatin.(Leishman 400x). 


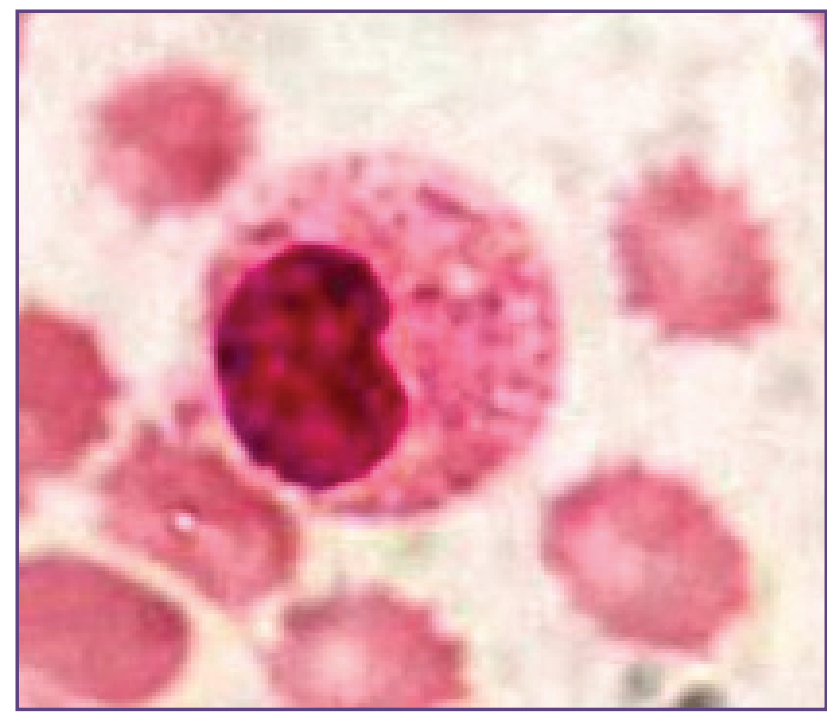

Fig. 2: Neutrophil showing intended unilobed nucleus with clumped chromatin.(Leishman 1000x) $\rightarrow$

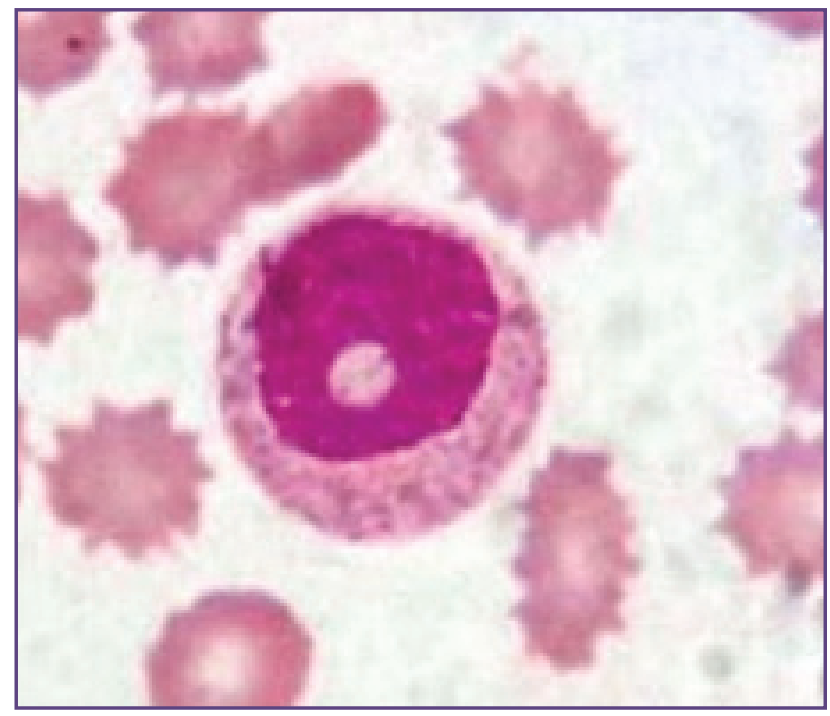

Fig. 4: Ring neutrophil.(Leishman 1000x).

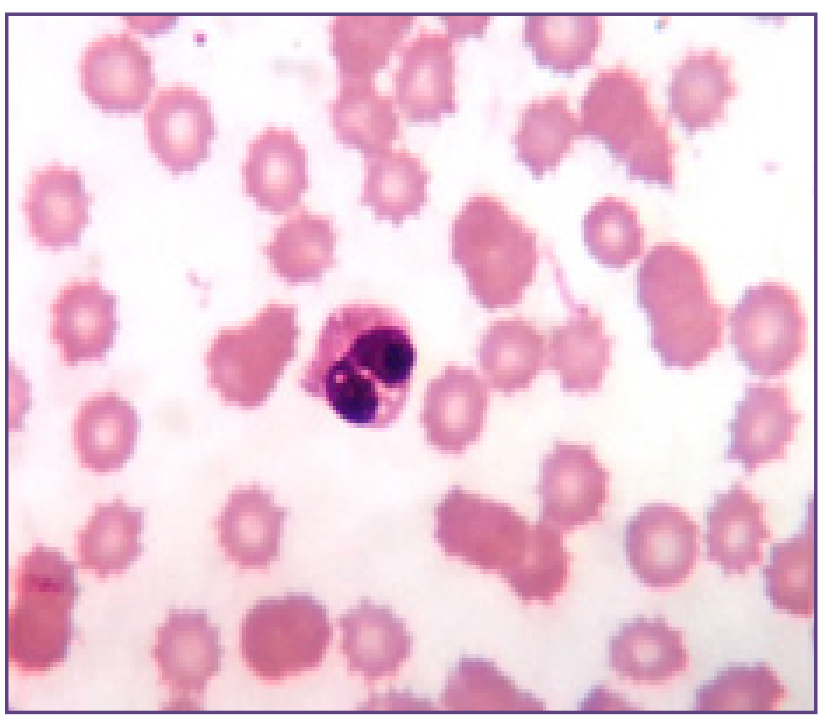

Fig. 3: Neutrophil showing bilobed nucleus with clumped chromatin.(Leishman 400x).

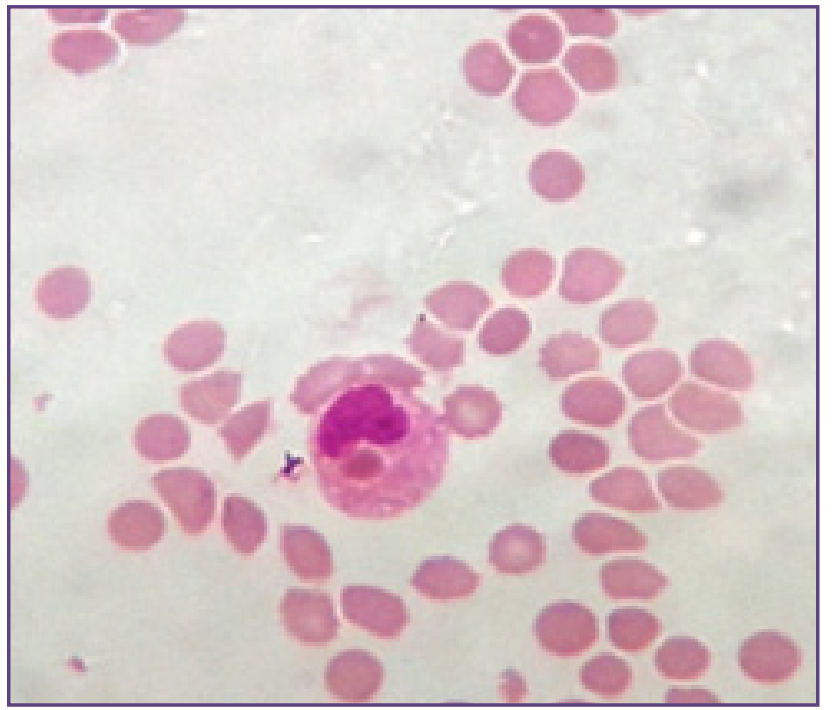

Fig. 5: Phagocytic cell engulfing an erythrocyte. (Leishman 1000x)

Table 1: Differential count of various morphology of the neutrophil.

\begin{tabular}{|l|c|}
\hline Morphology of the neutrophil & $\%$ \\
\hline Mature neutrophil & 5 \\
\hline Band form & 27 \\
\hline Bilobed neutrophil with clumped chromatin & 19 \\
\hline Unilobed neutrophil with clumped chromatin & 31 \\
\hline Unilobed intended neutrophil with clumped chromatin & 4 \\
\hline Ring neutrophil & 2 \\
\hline
\end{tabular}




\section{Discussion}

In 1928, Dr. Karl Pelger first described the "short and compact" neutrophil in two severely ill tuberculosis patients. He associated this peculiar neutrophil morphology for the poor prognosis and death of the patients. Later in 1932, Dr. G. J. Huet, A Dutch paediatrician, observed the same feature of the neutrophil in a 7 year old girl diagnosed with tuberculosis. To his surprise she recovered completely and screening of the child's close relatives showed the same hypolobulated neutrophil in some of their blood smears. So Heut discovered a genetic association with this condition with an autosomal dominant inheritance pattern and thus it was named as Pelger-Huet anomaly, later. ${ }^{[2,3]}$

Congenital Pelger-Huet Anomaly: PHA is an autosomal dominant condition with a prevalence of $0.01 \%$ to $0.1 \%$ in humans. It is also reported to be occurring in mammals like dogs and cats. ${ }^{[4]}$

Recent studies reveal that PHA occurs as result of quantitative defect in lamin B receptor (LBR) gene located in chromosome 1q41-43. LBR protein is located in the inner nuclear membrane and is responsible for binding of B-type lamins and heterochromatin. Thus an intact LBR with the intact microtubular network is essential for maintaining the shape and contour of the nucleus. ${ }^{[5]}$ The eleven different mutations occuring in LBR protein resulting in reduced expression is responsible for abnormal morphology of neutrophil in PHA. ${ }^{[4]}$ Yet their life span and function remains normal. ${ }^{[6,7]}$

In case of congenital PHA the percentage of polymorphs with PHA morphology is from 63\% to $93 \%$. The cytoplasm is normal .The nuclei is unilobed or bilobed with symmetrical lobes and having lower nuclear-cytoplasmic ratio, and a clumped, coarse and darkly stained chromatin. The chromatin clumping may occur in other granulocytes also with absence of cytopenias and chromosomal abnormalities. ${ }^{[8]}$ Screening of other family members with complete blood count and peripheral smear is advised.

Pseudo Pelger Huet Anomaly: Pseudo Pelger Heut cells occurs in acquired conditions like myelodysplasia (MDS), myeloproliferative disorders, acute myeloid leukemia, leukemoid reactions and use of certain medications like Ibuprofen, Valproate, Tacrolimus, Ganciclovir, Sulfisoxazole, Colchicine, D-Penicillamine. ${ }^{[8,9]}$

Different theories are suggested for the pathogenesis of pseudo-PHA. One study suggests an acquired mutation in LBR gene in the dysplastic neutrophil. ${ }^{[4]}$ Another study done on peripheral smear and bone marrow aspirates in MDS patients suggests that dysplastic neutrophil when observed under electron microscope resembled mature granulocyte undergoing apoptosis. ${ }^{[10]}$ This would explain the death of pseudo Pelger -Huet cell due to excess production of proapoptotic cytokines like TNF alpha and interferon gamma in the microenvironment of the bone marrow of MDS patients. ${ }^{[11]}$

Pseudo PHA is also found to occur in severe infections. Lee et al has reported a case of Human granulocytic anoplasmosis (HGA), a tick borne disease associated with pseudo PHA, in which the hematological presentations on peripheral smear resolved with doxycycline treatment. ${ }^{[12]}$.

In case of acquired PHA, cytoplasm of the neutrophil may be vacuoled, and the lobes of the nuclei are not symmetrical. There is hypogranulation of neutrophil and presence of cytopenias and anemia with cytogenetic abnormality. ${ }^{[8]}$

Variants of Pelger -Heut Cell: PHA unilobed - Single ovoid nucleus with dense, clumped chromatin with lower N/C ratio. This has a similar morphological appearance of a myelocyte, which can be differentiated with higher N/C ratio and open chromatin. ${ }^{[13]}$ PHA with indented nucleus has more dense chromatin than that 
of the metamyelocyte. Bilobed PHA - pincenez appearance with symmetrical lobes and condensed chromatin. Whereas pseudo-pelger cells have bilobed nucleus with asymmetrical lobes and is hypogranular. ${ }^{[13]}$

\section{Conclusion}

In conclusion, in the era of automation, a simple diagnostic test of a peripheral smear examination should not be neglected as it provides a better understanding of Pelger-Huet cells and helps in further management of the patient. When pelgeroid cells are seen in the peripheral smear, severe infections should be ruled out apart from hematological malignancies. A repeat smear study after control of infection or cessation of the drugs is advised.

\section{References}

1. Wang E, Boswell E, Siddiqi I, Lu CM, Sebastian S, Rehder C, et al. Pseudo-pelger-huet anomaly induced by medications: a clinicopathologic study in comparison with myelodysplastic syndrome-related pseudo-pelger-huet anomaly. Am J Clin Pathol 2011; 135:291-303.

2. Skendzel LP, Hoffman GC. The pelger anomaly of leukocytes: Forty-one cases in seven families. Am J Clin Pathol 1962; 37: 294-301.

3. Cunningham JM, Patnaik MM, Hammerschmidt DE, et al. Historical perspective and clinical implications of the Pelger-Huët cell. Am J Hematol 2009; 84:116-119.

4. Best S, Salvati F, Kallo J, et al. Lamin B-receptor mutations in Pelger-Huët anomaly. Br J Haematol. 2003 ; 123(3):542-4.
5. Hoffmann K, Dreger CK, Olins AL et al. Mutations in the gene encoding the lamin $\mathrm{B}$ receptor produce an altered nuclear morphology in granulocytes (Pelger-Huet anomaly). Nat Genet 2002;31: 410-414.

6. Oosterwijk JC, Mansour S, van Noort G. Congenital abnormalities reported in Pelger-Huët homozygosity as compared to Greenberg/HEM dysplasia: highly variable expression of allelic phenotypes. J Med Genet 2003; 40:937-41.

7. Borovik L, Modaff P, Waterham HR, et al. Pelgerhuet anomaly and a mild skeletal phenotype secondary to mutations in LBR. Am J Med Genet A 2013; 161 A:2066-73.

8. Guido D'Angelo. The Pelger-Huet Anomaly. From morphology to clinical suspicion. International Journal of Clinical Medicine Research 2014; 4:125-27.

9. Ayan MS. Pseudo Pelger-Huet abnormality: case report. Reactions. $2015 ; 1573: 137$

10. Shetty VT, Mundle SD, Raza A. Pseudo Pelger-Huet anomaly in myelodysplastic syndrome: Hyposegmented apoptotic neutrophil?. Blood 2001; 98: 1273-1275.

11. Economopoulou C, Pappa V, Kontsioti F, et al. Analysis of apoptosis regulatory genes expression in the bone marrow (BM) of adult de novo myelodysplastic syndromes (MDS). Leuk Res 2008; 32: 61-69.

12. Lee $\mathrm{S}$, Khankhanian $\mathrm{P}$, Salama $\mathrm{C}$ et al. Int $\mathrm{J}$ Hematol 2015;102(1): 129-33.

13. Colella R, Hollensead SC. Understanding and Recognizing the Pelger-Huët Anomaly. Am J Clin Pathol 2012;137(3):358-66.

*Corresponding author:

Dr Ayeesha Sithika T., 7, CLC works road, Chrompet, Chennai, Tamil Nadu, India 600115.

Phone: +91 9488621532

E-mail: dr.ayeesha1@gmail.com,

Date of Submission : 28.07.2016

Date of Acceptance : 21.11.2016

Financial or other Competing Interests: None.
Date of Publication : 19.02.2017 